\title{
Analyzing risk and performance using the multi-factor concept
}

\author{
Erik M. Vermeulen ${ }^{\text {a,b }}$, Jaap Spronk ${ }^{\mathrm{a}, *}{ }^{*}$, Nico van der Wijst ${ }^{\mathrm{b}}$ \\ a Erasmus University Rotterdam, Department of Finance, P.O. Box 1738, 3000 DR Rotterdam, The Netherlands \\ ${ }^{b}$ EIM Small Business Research and Consultancy, Zoetermeer, The Netherlands
}

Received November 1993; revised June 1995

\begin{abstract}
In this paper, we present a new model to analyze the risk and the expected level of firm performance. This model is based on the multi-factor approach to risk, in which unexpected performance is explained through sensitivities to unexpected changes of risk factors. Instead of using the multi-factor approach for the analysis of security portfolios, it is used to analyze performance measures of firms. In this paper the multi-factor approach is not only used to analyze risk, but also to analyze the expected level of performance. Furthermore, it is analyzed how instruments, as for instance projects, can be used to change the risk and the expected level of performance. An illustrative application in the field of finance is presented, although the model can also be applied in other areas.
\end{abstract}

Keywords: Finance; Investment analysis; Modelling; Risk analysis; Risk management

\section{Introduction}

This paper presents a model that can be used for risk analysis. In developing the model, the following criteria played an important role. The model should be intuitively appealing, should reveal what it is that makes the firm risky, and also provide a framework for managing risks.

This paper starts with a presentation of a view of the firm and its environment in Section 2. In this view, a firm's performance is related to unexpected changes of risk factors through sensitivities. This approach to risk is called the multi-factor approach and has already been applied many times. To provide some examples, Spronk and Van der Wijst (1987) were the first to use the approach for analyzing

\footnotetext{
${ }^{*}$ Corresponding author.
}

corporate risk. Berry, Burmeister and McElroy (1988) applied the method to analyze stock returns, Van Aalst et al. (1993) use it for asset liability matching for pension funds, Hallerbach (1994) applies the method to portfolio analysis, Goedhart and Spronk (1991) illustrate some applications in the field of financial planning, and LoCascio and Spronk (1992) investigate the Italian stock market making use of the multi-factor method.

Although our model is similar to the model for stock returns, it extends current literature in two ways. Firstly, performance measures of firms (in contrast with security portfolios) are analyzed. Secondly, apart from risk also expected performance is analyzed by means of the multi-factor method. Hence, both risk and the expected level of performance are explained through sensitivities by risk factors. Furthermore, the influence of instruments such as investment projects on the sensitivities is 
analyzed as well as the firm's risk profile and the expected level of performance.

The relation between the performance measure and the risk factors is formalized mathematically in Section 3. Since risk management not only involves analyzing the risks, but also changing them, Section 4 investigates the influence of instruments on performance.

In order to control risk the sensitivities should be changed by means of instruments. In Section 5 three different types of instruments are presented. In our opinion most instruments from practice belong to one of these types. The influence of instruments on risk is analyzed in Section 6. Making use of probability density functions, it is shown how the various instruments affect the sensitivities and what the consequences for the probability density function of unexpected performance are. Section 7 analyzes the influence of instruments on the expected level of performance. Section 8 shows how risk and the expected level of performance are traded-off in the multi-factor approach. This is illustrated by a numerical example in Section 9. Some conclusions are presented in Section 10.

\section{The firm and its environment: the main con- cepts}

A familiar view represents the firm as an input, throughput, output organization. The firm is assumed to use raw material and labor as inputs. These are transformed by the firm into various products and/or services, i.e., the firm's output. The inputs have to be paid for and, hence, lead to cash outflows, whereas the outputs generate cash inflows. The cash flows are defined as the sum of the cash inflows and cash outflows.

The supply of input factors and their prices, as well as the demand for output products and their prices are uncertain. Consequently, the cash inflows and cash outflows are not deterministic but stochastic. In other words, the firm faces a series of uncertainties.

Here, the analysis is restricted to the uncertainties that are caused by changes in risk factors, i.e., environmental stochasts that cannot be influenced by the firm. Examples are the wage rate, oil price and the exchange rate of the dollar. The risk factors influence the performance measures of the firm. The magnitude of this influence is called the sensitivity of the firm's performance measure to the risk factor. ${ }^{1}$

When analyzing the sensitivities, it is important to distinguish between expected and unexpected changes of the risk factors. Expected factor changes are foreseen and, thus, give the firm some time to react. Conversely, unexpected changes are not foreseen and the firm is less able to adapt to this new situation. Therefore, the sensitivity to an expected factor change will probably differ from the sensitivity to an unexpected factor change.

Unexpected changes in performance - i.e. the stochastic part of performance as opposed to the expected part - are the combined result of unexpected changes of the risk factors and the sensitivities to these changes. These sensitivities together can be seen as a way to describe the risks the firm faces and consequently as a multi-dimensional risk measure. The higher the sensitivities, the greater the impact of an unexpected risk factor change on performance will be, and the greater the risks the firm runs. ${ }^{2}$ The vector containing the sensitivities to the various risk factors is called the risk profile.

The sensitivities are related to the firm's characteristics. ${ }^{3}$ For instance, the interest rate sensitivity is highly dependent on the firm's level of debt, and the wage rate sensitivity depends, among other things, on the number of employees. Similarly, the business cycle sensitivity is influenced by the firm's product range, and so on.

After the firm's risk profile is determined, the firm's management may wish to change the sensitivities by means of instruments. Examples of such instruments range from changing the product range to changing a loan with variable interest rate for one with a fixed interest rate.

\footnotetext{
${ }^{1}$ The risk concept used here is largely in line with that of Cooper and Chapman $(1987$, p. 2) who define risk as: "exposure to the possibility of economic or financial loss or gain, physical damage or injury, or delay, as a consequence of the uncertainty associated with pursuing a particular course of action."

${ }^{2}$ Of course, the variance of the risk factors should also be taken into account when evaluating the risk profile.

${ }^{3}$ Actually, they may also depend on industry characteristics, such as the degree of competition in an industry.
} 


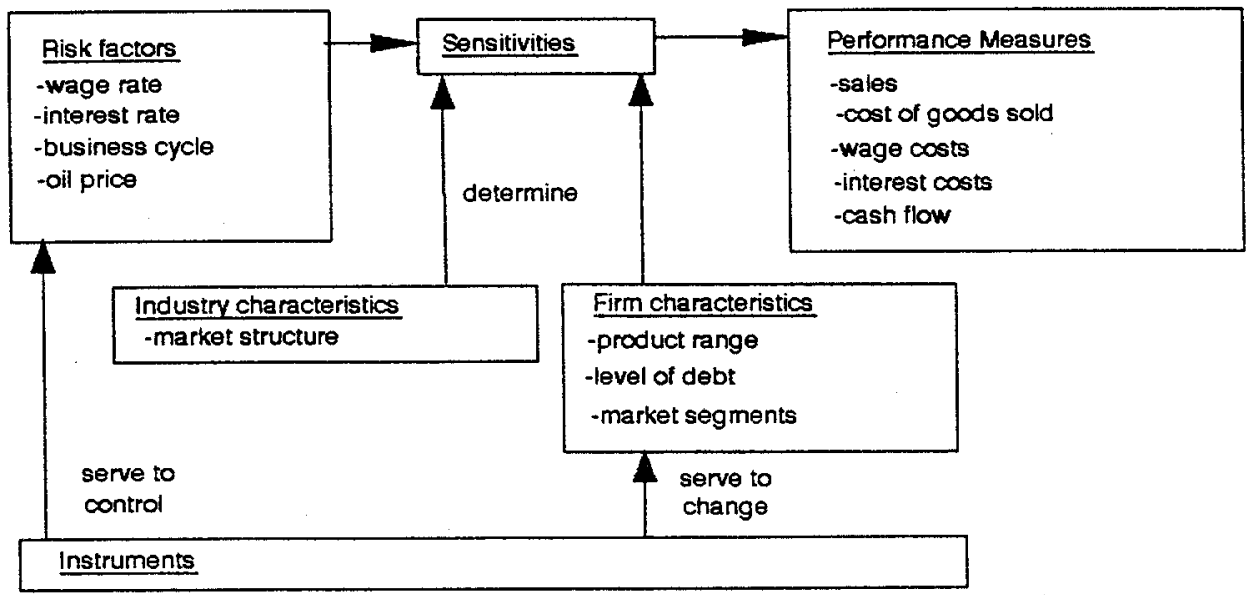

Fig. 1. The relation between risk factors, sensitivities, performance and instruments.

Finally, management has to decide whether or not to apply the instrument. The outcome of this decision process depends on the trade-off between an increase in return on the one hand, and a decrease in risks on the other.

The framework is summarized in Fig. 1. It is seen that risk factors affect performance through sensitivities, and that the sensitivities depend on both firm and industry characteristics. Furthermore the sensitivities can be changed by means of instruments. The instruments may influence the relation between the risk factors and performance. As will be seen in Section 5 this can be realized by either changing the firm characteristics or by controlling the risk factors.

This view on the firm and its environment will be used to analyze the risks of the firm's performance measures. The choice of the performance measure is free, and can range from the firm's number of employees to its "contribution"' to environmental pollution.

An advantage of this approach to risk analysis is that it is intuitively appealing. The uncertainties faced by the firm are related to risk factors with an economical meaning. As a result, it indicates what it is that makes the firm risky.

\section{The basic model}

In this section the view of the firm and its environment presented in Section 2 is formalized mathe- matically. For this purpose, a one-period model is constructed that can be used to analyze the risk and expected level of a firm's performance. ${ }^{4}$ The model will be restricted to one risk factor, but can be extended to more risk factors. ${ }^{5}$ The following relation between performance and the risk factor is assumed: ${ }^{6}$

$\tilde{R}_{t}=a_{t}+b_{t} \tilde{f}_{t}+\tilde{\eta}_{t}$

where

$\tilde{R}_{t}=$ performance,

\footnotetext{
${ }^{4}$ An advantage of the one-period model is that it facilitates easy presentation. Extension to more than one period is possible. However, this is more complex since in that case a trade-off has to be made between the risks and returns in the different periods. Since the main principles of risk management shown in the one period model are equal to those of the multi-period model, we shall concentrate on the one period model only.

${ }^{5}$ All ideas can easily and similarly be applied in the multi-factor case. The reason that we do not do so is that it only complicates notation without adding substantially to the understanding of the underlying ideas.

${ }^{6}$ This model differs from the arbitrage pricing theory (APT) as developed by Ross (1976) in several aspects. The APT describes the pricing of assets in a general equilibrium framework. The approach presented in this article limits itself to postulating a functional relation between a performance measure and a multitude of stochastic risk factors. The multi-factor approach does not assume a general equilibrium framework nor intends to derive market prices for the risk factors involved, see Hallerbach (1994, p. $33 \mathrm{ff})$.
} 
$a_{t}=\mathrm{a}$ fixed term,

$b_{t}=$ the sensitivity,

$f_{t}=$ the value of the risk factor,

$\tilde{\eta}_{t}=$ the error term,

$t=$ index for time,

$\tau=$ a random variable.

The fixed term $a_{t}$ and the sensitivity $b_{t}$ differ per firm, because of differences in firm characteristics, such as product range and management style.

From expression (1) the following expression is derived for performance at time $t+1$; defining $\Delta$ as the difference operator, i.e., $\Delta x_{t}=x_{t}-x_{t-1}$.

$$
\begin{aligned}
\tilde{R}_{t+1} & =a_{t+1}+b_{t+1} \tilde{f}_{t+1}+\tilde{\eta}_{t+1} \\
& =a_{t}+\Delta a_{t+1}+\left(b_{t}+\Delta b_{t+1}\right)\left(\tilde{f}_{t}+\Delta \tilde{f}_{t+1}\right) \\
& +\tilde{\eta}_{t+1},
\end{aligned}
$$

which, by assuming that the factor values at time $t$ have been realized, and thus are not stochastic anymore can be rewritten as:

$$
\begin{aligned}
\tilde{R}_{t+1}= & a_{t}+b_{t} f_{t}+\Delta a_{t+1}+\Delta b_{t+1} f_{t}+b_{t} \Delta \tilde{f}_{t+1} \\
& +\Delta b_{t+1} \Delta \hat{f}_{t+1}+\hat{\eta}_{t+1} .
\end{aligned}
$$

Apart from changes in risk factors, expression (2) leaves room for changes in the fixed term $a_{t}$ and in the sensitivity $b_{t}$. These changes are caused by instruments, which may vary from a change in firm characteristics to the purchase of a financial contract. The fixed term may change because of the (not factor related) costs associated with the application of the instruments.

In order to make a clear distinction between expected performance and unexpected performance, we split the change of the risk factor into an expected and an unexpected part.

$\Delta \hat{f_{t}}=\Delta \hat{f}_{\mathrm{U} t}+\Delta f_{\mathrm{E} t}$

where the subscripts $U$ and $E$ respectively denote unexpected and expected variables,

$\Delta \tilde{f}_{\mathrm{U} t}=\tilde{f}_{t}-\mathrm{E}\left(\tilde{f}_{t}\right)$,

$\Delta \tilde{f}_{\mathrm{E} t}=\mathrm{E}\left(\tilde{f_{t}}\right)-f_{t-1}$,

$\mathrm{E}=$ the expectation operator.
Substituting (3) into (2) leads, after rewriting, to the following expression:

$$
\begin{aligned}
\tilde{R}_{t+1}= & a_{t}+b_{t} f_{t}+\Delta a_{t+1}+\Delta b_{t+1} f_{t} \\
& +b_{t} \Delta f_{\mathrm{E} t+1}+\Delta b_{t+1} \Delta f_{\mathrm{E} t+1} \\
& +b_{t} \Delta \tilde{f}_{\mathrm{U} t+1}+\Delta b_{t+1} \Delta \tilde{f}_{\mathrm{U} t+1}+\tilde{\eta}_{t+1} .
\end{aligned}
$$

It can be seen that the first six elements concern the expected level of performance, whereas the last three elements refer to the risk of performance.

Furthermore, we make a distinction between the sensitivities to the factor level, unexpected factor change and the expected factor change. We also assume that the sensitivities are not equal to each other. This can be justified economically as was seen in Section 2.

$$
\begin{aligned}
\tilde{R}_{t+1}= & a_{t}+b_{\mathrm{L} t} f_{t}+\Delta a_{t+1}+\Delta b_{\mathrm{L} t+1} f_{t} \\
& +b_{\mathrm{E} t} \Delta f_{\mathrm{E} t+1}+\Delta b_{\mathrm{E} t+1} \Delta f_{\mathrm{E} t+1} \\
& +b_{\mathrm{U} t} \Delta \tilde{f}_{\mathrm{U} t+1}+\Delta b_{\mathrm{U} t+1} \Delta \tilde{f}_{\mathrm{U} t+1}+\tilde{\eta}_{t+1},
\end{aligned}
$$

where

$b_{\mathrm{L} t}=$ the sensitivity to the level of the risk factor,

$b_{\mathrm{E} t}=$ the sensitivity to the expected change of the risk factor,

$b_{\mathrm{U} t}=$ the sensitivity to the unexpected change of the risk factor.

A further extension is possible by assuming that $b_{\mathrm{E} t}$ and $b_{\mathrm{U} t}$ are not the same for all factor changes, but actually depend on these factor changes. In order to make this relation more explicit, we sometimes write $b_{\mathrm{E} t}\left(\Delta f_{\mathrm{E} t+1}\right)$ and $b_{\mathrm{U} t}\left(\Delta f_{\mathrm{U} t+1}\right)$ instead of $b_{\mathrm{E} t}$ and $b_{\mathrm{U} t}$.

It is not hard to imagine that in reality the sensitivity depends on the factor change. Take, for example, an increase in demand for the firm's product. Initially, production can be increased easily until maximum capacity is reached. After that, production can be increased at high costs, e.g. by working overtime, buying the necessary raw material from other suppliers, and lending money at a higher interest rate. The consequences for the sensitivity of the firm's cash flow to demand are that the cash flow sensitivity is a declining function of demand. 
As mentioned in Section 2 the sensitivities may also depend on the firm's characteristics. Mathematically, this can be written as $b_{\mathrm{U} t}\left(\mathrm{fc}_{t}\right)$, where $\mathrm{fc}_{t}$ denote the firm characteristics.

\section{The change in performance and the influence of instruments}

Using expression (5) the impact of instruments on both risk and the expected level of performance can be analyzed. For this purpose, we simply have to compare performance with and without instruments.

Should no instruments be used, then the sensitivities as well as the fixed term are constant, thus $\Delta a_{t+1}=\Delta b_{\mathrm{L} t+1}=\Delta b_{\mathrm{E} t+1}=\Delta b_{\mathrm{U} t+1}=0$ and performance at $t+1$ is:

$\tilde{R}_{t+1}=a_{t}+b_{\mathrm{L} t} f_{t}+b_{\mathrm{E} t} \Delta f_{\mathrm{E} t+1}+b_{\mathrm{U} t} \Delta \tilde{f}_{\mathrm{U} t+1}+\tilde{\eta}_{t+1}$,

This relation, which can easily be extended to more than one factor ${ }^{7}$, is more extensive than the relation generally found in finance textbooks, e.g. Ross et al. (1993, p. 322). In expression (6) we explicitly take account of the sensitivity to the expected factor change and the factor level. The reason for this is that our multi-factor model is intended to be used to analyze financial flows in the firm, whereas the purpose of most multi-factor models is to analyze stock returns. From a theoretical point of view, the sensitivity of stock returns to expected factor changes should be zero, since the influence of expected factor changes is assumed to be absorbed immediately in the stock price. Consequently, the expected factor change can be left out of consideration in those models. ${ }^{8}$

When analyzing other performance measures, the extension is important since in that case expected factor changes often do not lead to different performance patterns until their realization.

\footnotetext{
${ }^{7}$ See also Footnote 5.

${ }^{8}$ In contrast to expected factor changes, unexpected factor changes influence the stock price. Accordingly, the sensitivity to unexpected factor changes is not necessarily equal to zero. Finally, stock prices may also change as a result of a change in the stock's sensitivity.
}

Subtracting expression (6) from expression (5) reveals the influence of instruments. ${ }^{\text {? }}$

$$
\begin{aligned}
I \tilde{R}_{t+1}= & \Delta a_{t+1}+\Delta b_{\mathrm{L} t+1} f_{t}+\Delta b_{\mathrm{E} t+1} \Delta f_{\mathrm{E} t+1} \\
& +\Delta b_{\mathrm{U} t+1} \Delta \tilde{f}_{\mathrm{U} t+1},
\end{aligned}
$$

where

$\tilde{I I R}_{t+1}=$ the influence of instruments on performance at time $t+1$.

The first three terms of this expression refer to the influence of instruments on expected performance, ${ }^{10}$ the last term to their influence on risk.

Expression (7) can also be used to clarify the difference between real and financial instruments. ${ }^{11}$ Financial instruments often have to be paid for and solely reduce uncertainty, i.e., they solely affect $\Delta a_{t}$ and $\Delta b_{\mathrm{U} t}$. Real instruments, however, have to be paid for and influence the sensitivity to the level of the risk factor, the expected and the unexpected factor change, hence they influence $\Delta a_{t}, \Delta b_{\mathrm{L} t}, \Delta b_{\mathrm{E} t}$ and $\Delta b_{\mathrm{U} t}$.

\section{Instruments that change the sensitivities}

Starting point of the analysis of the influence of instruments on performance at time $t+1$ is expression (7). As far as the expected level of performance is concerned, instruments affect the first three terms of expression (7): the constant term, the sensitivity to

\footnotetext{
${ }^{9}$ Using the same method, the change of performance from time $t$ to $t+1$ when instruments are used can be obtained by subtracting performance at time $t$, i.e. expression (1), from performance at time $t+1$ when instruments are used, i.e. expression (5) and so on.

${ }^{10}$ The terms "expected performance" and "expected level of performance" are interchangeable.

${ }^{11}$ Shapiro and Titman (1992, p. 341) make a distinction between financial and real instruments: "The principal risk-reducing techniques that can be categorized as financial include lowering the firm's debt-equity ratio, buying or selling forward or futures contracts, and buying insurance.. Real adjustments include the adoption of production processes that reduce the degree of operating leverage, avoidance of high-risk projects, and abandonment of existing high risk products..." It will be clear that those instruments that are classified as real are likely to affect both the sensitivities to expected and unexpected risk factor changes, whereas those classified as financial only affect the sensitivities to unexpected factor changes.
} 
the factor level and the sensitivity to the expected change of the risk factor. As regards risk, they affect the last term denoting the sensitivity to the unexpected change of the risk factor.

Three different kinds of instruments are distinguished in this paper. The first kind of instruments change the level of the sensitivity at time $t$, and are called level changing instruments. When a level changing instrument is applied the sensitivity change does not depend on the factor value. As a result, application of a level changing instrument leads to either an increase of the sensitivity to both positive and negative risks, or to a decrease of the sensitivity to these risks. The change in level can be denoted as $\Delta b_{t}=c_{1} .{ }^{12}$ Take a change in number of employees as an example of a level changing instrument. This change will probably affect the sensitivity to the wage rate.

Another kind of instruments are flexibility instruments. A characteristic of flexibility instruments is that they make the sensitivity dependent on the realization of the unexpected factor change. Therefore, the effects of flexibility instruments are denoted as $\Delta b_{t}=c_{\mathrm{f}} \cdot \mathrm{e}\left(\Delta \tilde{f}_{\mathrm{U} t}\right)$. The symbol $\mathrm{e}\left(\Delta \tilde{f}_{\mathrm{U} t}\right)$ indicates that the sensitivity depends on the factor change. The symbol $c_{\mathrm{f}}$ is used for scaling purposes. As an example take a machinery that can be used to make different products, so that the different demand in various stages of the business cycle can be met. Should the business cycle increase, it will be advantageous to change production to more luxurious and profitable products in order to benefit from the rise of the business cycle. In the opposite case it will be sensible to shift away from those luxurious products. Thus, it is clear that flexibility increases the possibilities to benefit from positive risks and to avert negative risks.

One important difference between level changing instruments and flexibility instruments is that the latter can be used to react to unexpected factor changes, whereas the decision to apply the first has to be made in advance, i.e. before the realization of the unexpected factor change.

The last category of instruments are transforma-

\footnotetext{
${ }^{12}$ In this section, $\Delta b_{t}$ is used to denote $\Delta b_{\mathrm{L}_{\ell}}, \Delta b_{\mathrm{E} t}$, and $\Delta b_{\mathrm{U} t}$.
}

tion instruments, which transform the unexpected change of the risk factor. Transformation instruments actually control the risk factor in order to keep the risks manageable. Their effect is denoted as $\Delta b_{t}=$ $c_{t \mathrm{r}} \cdot \mathrm{T}\left(\Delta \tilde{f}_{\mathrm{U} t}\right) / \Delta \tilde{f}_{\mathrm{U} t}$. Again, $c_{\mathrm{tr}}$ is used for scaling purposes. The symbol $\mathrm{T}\left(\Delta \tilde{f}_{\mathrm{U} t}\right)$ denotes that the factor change is transformed. The ratio $\mathrm{T}\left(\Delta \tilde{f}_{\mathrm{U} t}\right) / \Delta \tilde{f}_{\mathrm{U} t}$ is used, because the transformed factor change should be related to the actual factor change. A simple example of transformation instruments is a cap. The cap transforms the unexpected changes of the risk factor: all realized values of the risk factor below the level of the cap remain unchanged, whereas those exceeding it are limited by the level determined by the cap. For instance, an interest rate cap of $7 \%$ guarantees the buyer of the cap that he pays the variable interest rate on the amount borrowed if the rate is lower than $7 \%$, whereas only $7 \%$ interest has to be paid if the rate exceeds $7 \%$. Accordingly, interest rate caps can be used to reduce the risk of a rise in the interest rate.

In order to make the instrument concept more concrete, Table 1 presents some examples of instruments from practice. As a first example of a level changing instrument a change in production level is mentioned. Since such a change similarly affects the consequences of positive and negative risks, it is called a level changing instrument. Similar arguments hold for transferring input prices and avoiding high risk projects.

As examples of flexibility instruments, all contingent claims the firm has can be mentioned. Risk is managed by keeping flexible, which enables the firm to react upon the realization of the unexpected factor

Table 1

Some practical instruments and their classification

Level changing instruments
Changing the firm's production level; Passing on input price
changes to customers; Avoiding high risk projects.

Flexibility instruments

Options; Shut down options; Abandonment options; Changing machine options; Option to dismiss employees.

Transformation instruments

Caps /floors; Swaps; Futures /Forwards; Insurances; Fixed price contracts; Fixed volume contracts. 
changes. For instance, in case the demand for a factory's product turns out to be far too low, a shut down option may be valuable. In the finance literature, these options are referred to as real options.

Examples of transformation instruments, actually are all financial contracts that serve to transform the future values of the risk factors. Thus, all fixed price contracts, minimum delivery contracts and so on can be seen as examples of them.

\section{The impact of instruments on risk}

In this section the influence of instruments on the sensitivity to the unexpected change in the risk factor, $\Delta b_{\mathrm{U} t+1}$, will be discussed. Probability theory will be used to investigate the way in which risk is changed. First, the probability density function of unexpected, factor related performance ${ }^{13}$ is assumed to depend through sensitivities on the probability density functions of the risk factors. Then, it is investigated how changing the sensitivity by means of the various kinds of instruments discussed above, affects the probability density function of unexpected, factor related, performance. Finally, two techniques to reduce risks will be discussed. The analysis will be illustrated by an example.

In this example we use a one-period model from time $t$ to $t+1$. We assume that the interest rate is the only risk factor, that its expected value at $t+1$ is $6 \%$, and that the probability density function (p.d.f.) of an unexpected change in the interest rate at $t+1$ follows a normal distribution. This distribution is represented by the graph in Fig. 2. Furthermore, suppose someone borrowed $\$ 100,000$.- at the variable interest rate described above. Accordingly, the sensitivity, " $b_{\mathrm{U}} t$ ", has the value of $-(\$ 1000 / 1 \%)$, which implies that the change in interest costs is $\$ 1000$ per one percent change in the interest rate. The sensitivity is independent of the unexpected change of the interest rate. This sensitivity is shown in the upper picture of Fig. 3. The middle picture

\footnotetext{
${ }^{13}$ The term unexpected, factor related, performance refers to that part of unexpected performance that is related to unexpected changes of the risk factors. In the present analysis the residual error term, $\eta_{t+1}$, is left out of consideration.
}

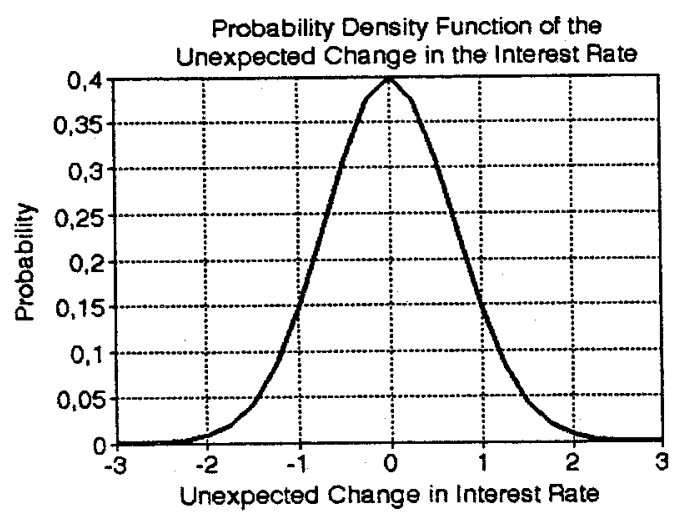

Fig. 2. The probability density function of the unexpected change in the interest rate.

presents the relation between the unexpected, factor related, change of performance and the unexpected change of the interest rate - i.e. in symbols, the relation between $b_{\mathrm{U} t} \Delta \tilde{f}_{\mathrm{U} t+1}$ and $\Delta \tilde{f}_{\mathrm{U} t+1}$. The lower picture displays the corresponding p.d.f. of the unexpected, factor related, change of performance, which can be obtained from the middle picture and the p.d.f. of the unexpected change of the interest rate.

Let us now investigate the influence of the three kinds of instruments on both the sensitivity and the unexpected, factor related, change of performance. Suppose that the borrower agrees to pay a fixed interest rate on the first $\$ 40,000$.- borrowed. In fact, this can be seen as the application of a level changing instrument with $\Delta b_{\mathrm{U} t+1}=c_{1}=\$ 400 / 1 \%$. As a result, the sensitivity is increased from $-\$ 1000 / 1 \%$ to $-\$ 600 / 1 \%$, see the upper picture of Fig. 4 . It is clear from the middle picture that decreasing the absolute value of the level of the sensitivity makes the linear relation between the unexpected, factor related, change of performance and the unexpected change of the risk factor less steep, and leads to a reduction of the variance of unexpected performance (Fig. 4, lower picture). It is seen that both positive and negative risks are reduced. An unexpected increase in the interest rate will be less harmful (than without application of the instrument). On the other hand, the firm will also benefit less from a drop in the interest rate.

To illustrate how flexibility instruments work, it is assumed that the sensitivity can be adapted to the realized value of the unexpected change of the risk 

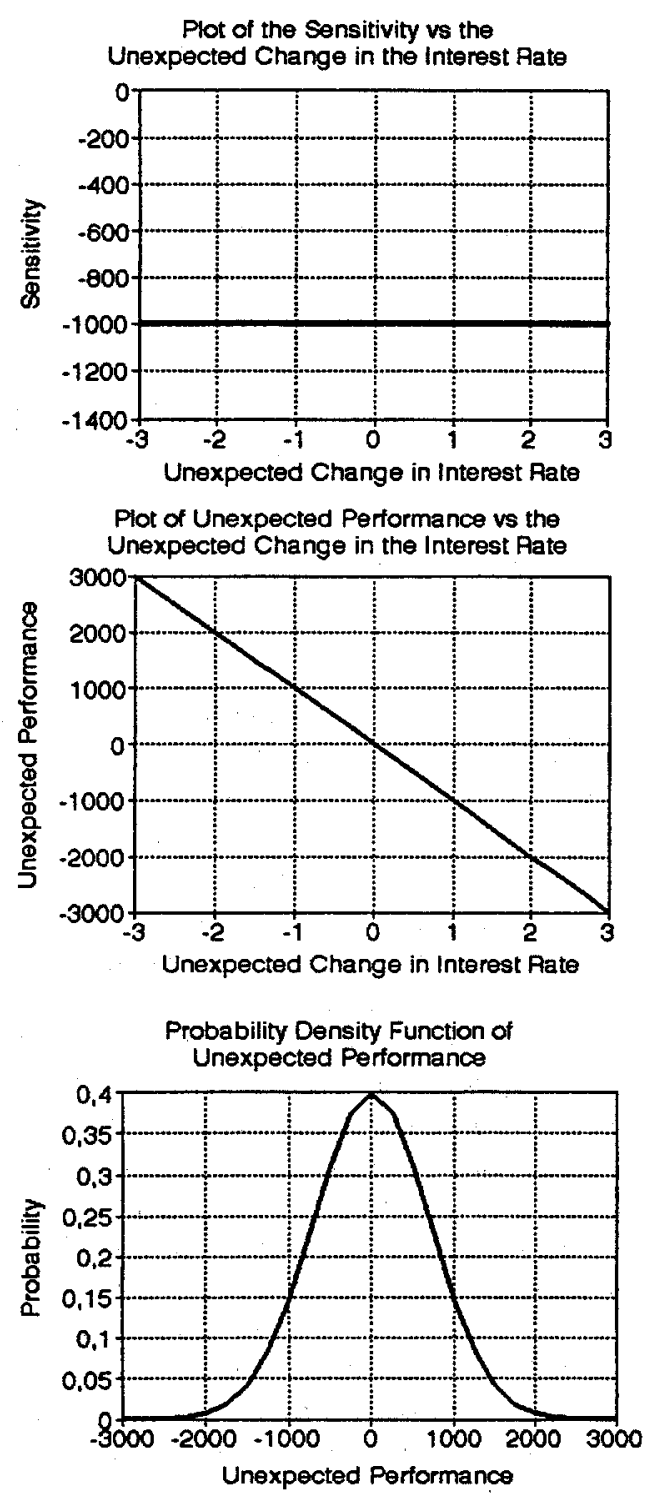

Fig. 3. Without instruments.

factor at $t+1$ and, consequently, is increased (as opposed to the level of the sensitivity at time $t$ ) in case of a positive change of the risk factor (Fig. 5, upper picture). In this case, $c_{\mathrm{f}}=\$ 800 / 1 \%$, and $\mathrm{e}\left(\Delta \tilde{f}_{\mathrm{U} t+1}\right)=1$ if $\Delta \tilde{f}_{\mathrm{U} t+1}>0$, and $\mathrm{e}\left(\Delta \tilde{f}_{\mathrm{U} t+1}\right)=0$ if $\Delta \tilde{f}_{\mathrm{U} t+1} \leq 0$. The corresponding sensitivity equals $b_{\mathrm{U} t+1}+\Delta b_{\mathrm{U} t+1}=-\$ 200 / 1 \%$ if $\Delta \tilde{f}_{\mathrm{U} t+1}>0$, and $-\$ 1000 / 1 \%$ if $\Delta \tilde{f}_{\mathrm{U} t+1} \leq 0$. The consequences of application of this flexibility instrument are that the linear relation between the unexpected, factor related, change of performance and the unexpected change of the risk factor is split (Fig. 5, middle picture), and the p.d.f. of unexpected, factor related, change of performance is skewed to the right (Fig. 5, lower picture), thus increasing the possibility of higher unexpected, factor related, change of perfor-
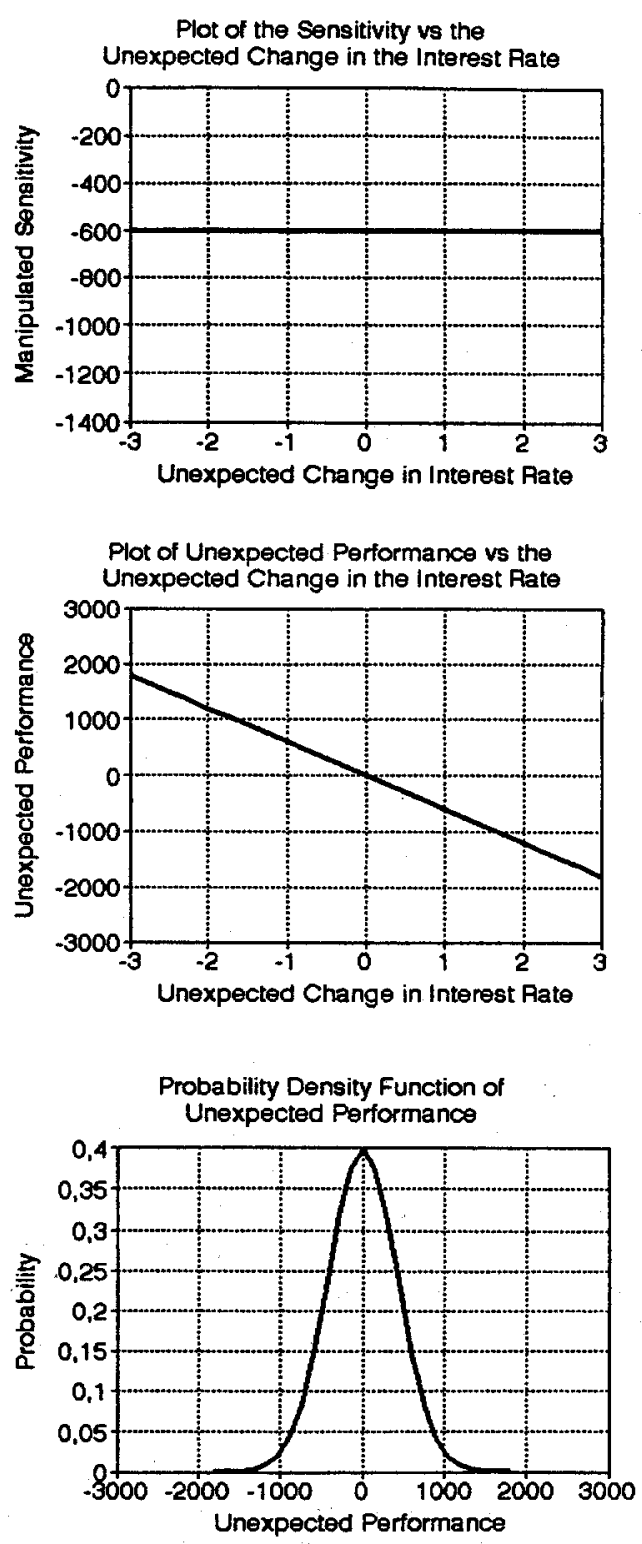

Fig. 4. The influence of a level changing instrument. 

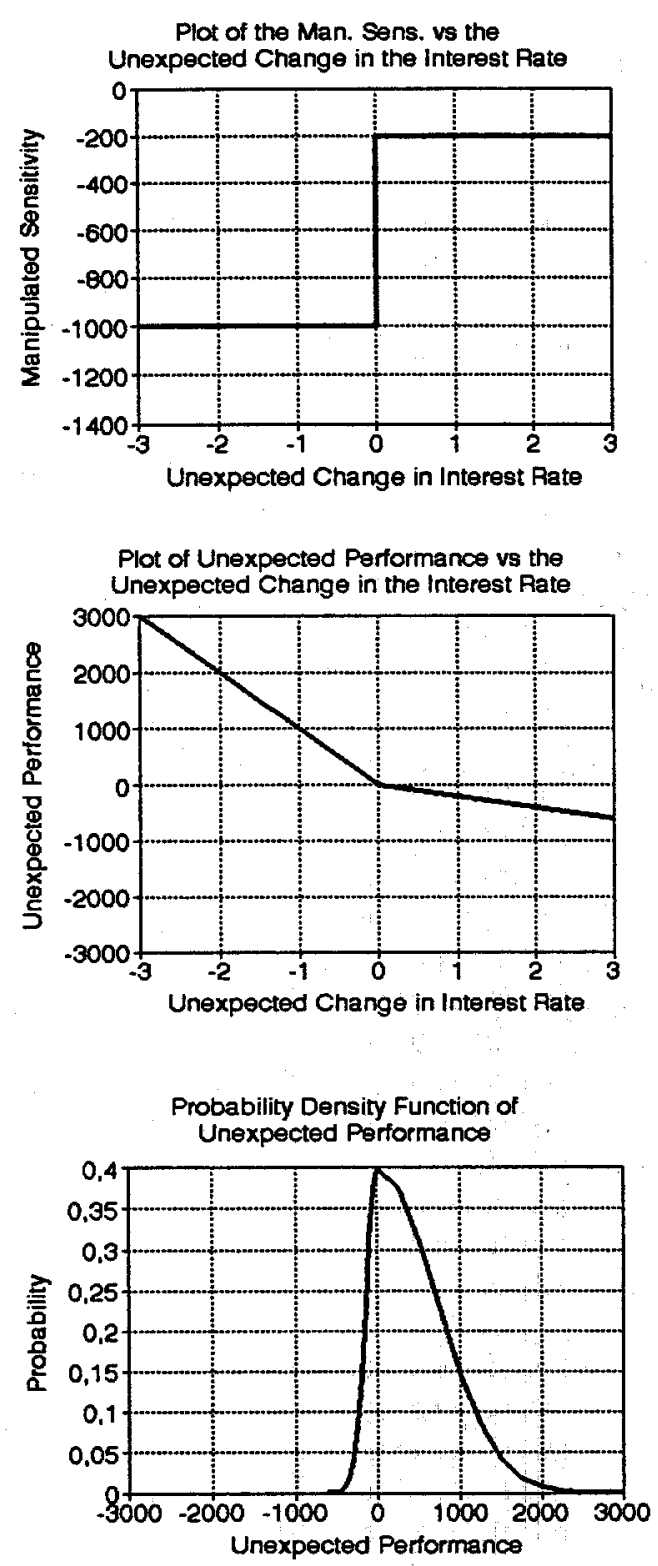

Fig. 5. The influence of a flexibility instrument.

mance and decreasing the probability of a loss. Evidently, flexibility instruments avert negative risks, while enabling the firm to profit from positive risks.

To clarify the influence of transformation instruments assume that a cap at $7 \%$ has been bought, i.e. a contract is made to ensure that the current interest rate has to be paid for all interest rates lower than or equal to $7 \%$, but for all interest rates higher than $7 \%$ an interest rate of only $7 \%$ has to be paid. The corresponding transformation function is: $c_{\mathrm{tr}}=$ $\$ 1000 / 1 \%$, and $\mathrm{T}\left(\Delta f_{\mathrm{U} t+1}\right) / \Delta f_{\mathrm{U} t+1}=0$ if $\Delta f_{\mathrm{U} t+1}$ $<(7 \%-6 \%)=1 \%$, and $\mathrm{T}\left(\Delta f_{\mathrm{U} t+1}\right)=(1-$ $\left.\left(1 / \Delta f_{\mathrm{U} t+1}\right)\right)$ if $\Delta f_{\mathrm{U} t+1} \geq 1 \%$. Accordingly, the sensitivity equals $b_{\mathrm{U} t}+\Delta b_{\mathrm{U} t+1}=\$ 1000 / 1 \%$ if $\Delta f_{\mathrm{U} t+1}<1 \%$, and $-\$ 1000 / \Delta f_{\mathrm{U} t+1}$ if $\Delta f_{\mathrm{U} t+1} \geq$
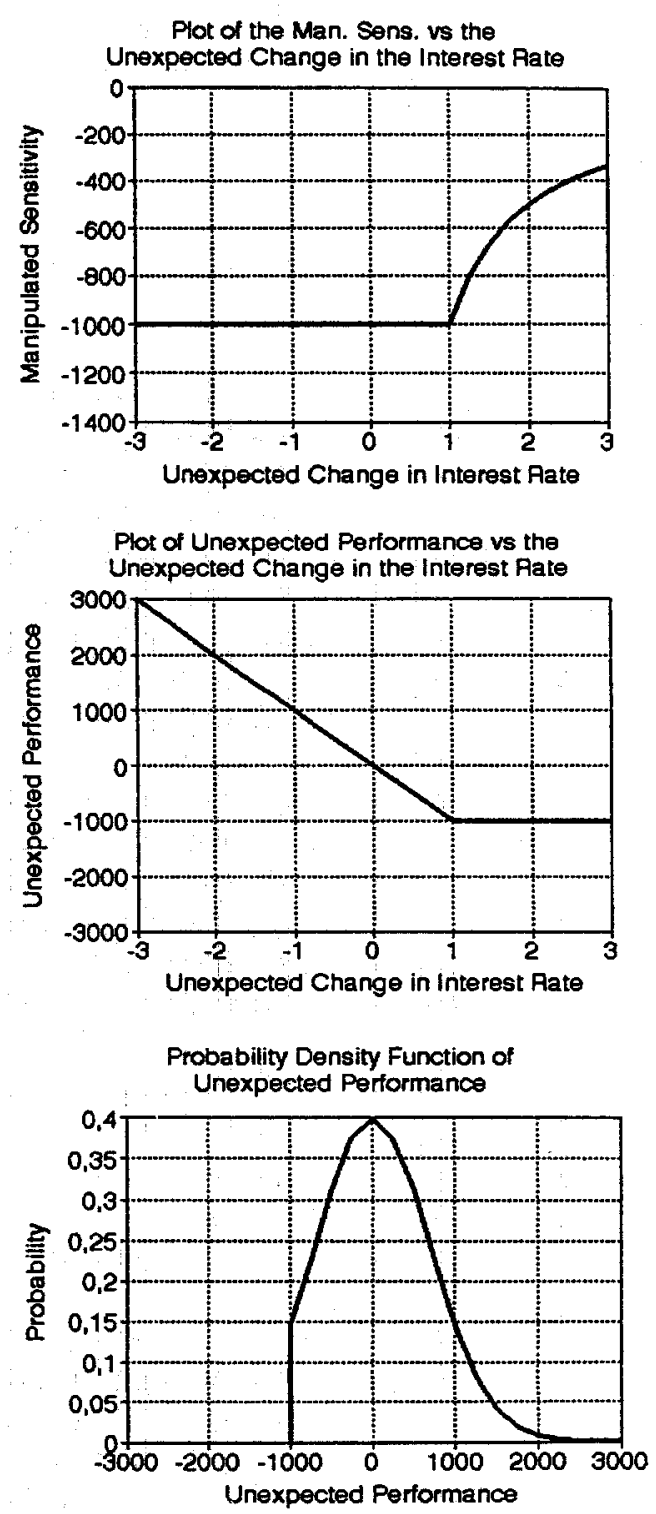

Fig. 6. The influence of a transformation instrument. 
$1 \%$. It is seen that, in case of changes of more than $1 \%$, the absolute value of the sensitivity gradually decreases (see the upper picture of Fig. 6), and that the unexpected, factor related, change of performance as a function of the unexpected change of the risk factor becomes constant (middle picture). The result is that the p.d.f. of unexpected, factor related, change of performance is truncated (lower picture). It should be stated that the probability of unexpected performance can also adapt other shapes when transformation instruments are applied. For instance, were the risk factor transformed by closing a contract with fixed interest rate, then uncertainty in unexpected performance would be reduced completely.

\section{The impact of instruments on the expected level of performance}

As far as the expected level of performance is concerned, instruments can influence the fixed term, $\Delta a_{t+1}$, the sensitivity to the level of the risk factor, $\Delta b_{\mathrm{I} t+1}$, and the sensitivity to the expected change of the risk factor $\Delta b_{\mathrm{E}_{t+1}}$. As mentioned before, $\Delta a_{t+1}$ represents a not factor related change in performance. An example of $\Delta a_{t}$ are the costs to be paid for an instrument that changes the sensitivity.

It is easier to analyze the influence of instruments on $\Delta b_{\mathrm{L} t+1}$ and $\Delta b_{\mathrm{E} t+1}$ than on $\Delta b_{\mathrm{U} t+1}$, because $f_{\mathrm{U} t}$ and $\Delta f_{\mathrm{E} t}$ are non-stochastic in contrast to $\Delta f_{\mathrm{U} t+1}$. As a result, the consequences for the various probability density functions do not have to be analyzed, and only the value of the function describing the instrument has to be computed given the level of the factor value and/or the expected change of the factor value.

The analyses are simple. Level changing instruments cause $\Delta b_{\mathrm{E} t+1}$ to become $c_{\mathrm{t}}$. Flexibility instruments cause $\Delta b_{\mathrm{E} t+1}$ to become $c_{\mathrm{f}} \cdot \mathrm{e}\left(\Delta f_{\mathrm{E} t+1}\right)$. Transformation instruments cause $\Delta b_{\mathrm{E} t+1}$ to become $\left.c_{\mathrm{t}} \cdot \mathrm{T}\left(\Delta f_{\mathrm{E} t+1}\right) / \Delta f_{\mathrm{E} t+1}\right)$. The same holds for $\Delta b_{\mathrm{L} t+1}$.

Especially when real instruments are applied to reduce risks, the consequences of instruments for the expected level of performance may be large. After all, real instruments, such as a production shift, not only affect the sensitivity to the unexpected factor change, but also are likely to affect the level of the sensitivity and the sensitivity to the expected factor change.

\section{The trade-off between risk and the expected level of performance}

After the analysis of the influence of instruments on risk and the expected level of performance, the question becomes how the decision maker can choose between the different alternatives. The answer depends on the set of alternatives and on the information available on the decision maker's preferences. Furthermore, it depends on the possible market prices relating to the risk factors. Examples of priced risk factors are the oil price and the interest rate.

However, there may also be risk factors which are not priced and which are nevertheless important to the decision maker. Here one can think of the wage rate and the business cycle. In these cases the decision maker should make a trade-off between the risks and returns of the different alternatives. One may sometimes assume that a Multi-Attribute Utility Function can be used to describe the decision maker's preferences. The choice between different alternatives then becomes one of choosing the alternative with the highest expected utility (see e.g. Keeney and Raiffa, 1976).

Sometimes it suffices to assume that the decision makers are utility maximizing and risk averse. These decision makers can be shown to:

- always apply an instrument if it increases expected performance and decreases risk;

- never apply an instrument that decreases expected performance and increases risk;

- make a trade-off if both risk and expected performance either increase or decrease simultaneously. In our framework, risk is reduced when (Vermeulen, 1994)

$\left|b_{\mathrm{U} t}+\Delta b_{\mathrm{U} t+1}\right|<\left|b_{\mathrm{U} t}\right|$.

Thus, a reduction in risk implies a decrease in the value of a positive sensitivity and an increase in the value of a negative sensitivity. This situation is also shown in Figs. 3 and 4.

Expected performance is increased when

$\Delta a_{t+1}+\Delta b_{\mathrm{L} t+1} f_{t}+\Delta b_{\mathrm{E} t+1} \Delta f_{\mathrm{E} t+1}>0$. 
In many practical cases $\Delta b_{\mathrm{L} t+1}, \Delta b_{\mathrm{E} t+1}$, and $\Delta b_{\mathrm{U} t+1}$ may have nearly the same values. Consequently, a change in expected performance often accompanies a change in risk.

Since the method presented in this paper is applied to a multitude of risk factors trade-offs between the increase in expected performance on the one hand and the sensitivities to the various risk factors should be made. This situation becomes much more complicated and it will often be hard if not impossible to define a reliable multi-attribute-utility function. In such case, an abundance of multiple criteria decision methods exists.

\section{An illustration}

The trade-off mentioned in Section 8 will be illustrated here by a numerical example. The analysis consists of first considering the influence of a replacement decision on the expected level of performance, then on risk, and finally on the trade-off between these two. All relevant terms concerning the analysis can be found in expression (7), which is repeated as:

$$
\begin{aligned}
I \tilde{I R}_{t+1}= & \Delta a_{t+1}+\Delta b_{\mathrm{L} t+1} f_{t}+\Delta b_{\mathrm{E} t+1} \Delta f_{\mathrm{E} t+1} \\
& +\Delta b_{\mathrm{U} t+1} \Delta \tilde{f}_{\mathrm{U} t+1} .
\end{aligned}
$$

Assume that a car factory's management wishes to reduce its energy price sensitivity by buying more efficient machines. Suppose that changing the old machines for new ones costs $\$ 100,000$, assume furthermore that the new machines use 2 million kilowatt hours (hereafter: $\mathrm{kWh}$ ) less energy. The consequences of this decision are as follows. The fixed costs connected to the decision are $\Delta a_{t+1}=$ $-\$ 100,000$. Secondly, we assume that the change in sensitivity to the factor level, the expected factor change and the unexpected factor change are equal to each other, thus $\Delta b_{\mathrm{U} t+1}=\Delta b_{\mathrm{E} t+1}=\Delta b_{\mathrm{L} t+1}=$ $\Delta b_{t+1}=2$ million $\mathrm{kWh}$. Hence, $\Delta b_{\mathrm{L} t+1} \cdot f_{\mathrm{t}}+$ $\Delta b_{\mathrm{E} t+1} \cdot \Delta f_{\mathrm{E} t+1}=\Delta b_{t+1} \cdot f_{\mathrm{E} t}=2$ million $* f_{\mathrm{E} t+1}$. Assume that the expected energy price is $\$ 0.15$ per $\mathbf{k W h}$, then replacement leads to an advantage of 2 million $* \$ 0.15=\$ 300,000$. On balance, replacement increases expected performance by $\$ 300,000-$ $\$ 100,000=\$ 200,000$.
As regards risk, the negative sensitivity to unexpected changes in the energy price will decrease by $\Delta b_{\mathrm{U} t+1}=2$ million $\mathrm{kWh}$ as well. This change in the level of the sensitivity will cause a decrease in the variance of unexpected performance (refer to Figs. 3 and 4 for an illustration).

The conclusion is that given this analysis the firm should replace old machines by new ones, since this change implies an increase in expected performance and a decrease in risk as far as the sensitivity to the energy price is concerned.

\section{Conclusion and discussion}

In this paper we presented a framework for analyzing the influence of instruments on risk and the expected level of performance. For this purpose we first developed a basic model that relates the firm's performance to risk factors through sensitivities.

Next, three kinds of instruments were discussed that can be used to change the sensitivities: level changing instruments, flexibility instruments and transformation instruments. We investigated the influence of the various kinds of instruments on performance by analyzing their impact on the probability density function of performance. It appeared that level changing instruments change the variance of the probability density function of performance influencing the consequences of both positive and negative risks in the same direction. Flexibility instruments distort this probability density function, enabling the firm to take advantage of positive risks and to avert negative risks. The influence of transformation instruments on the probability distribution of unexpected performance depends heavily on the kind of transformation instrument used.

Furthermore, the influence of instruments on expected performance was analyzed. Especially for real instruments, the effect on the sensitivity to the risk factor level and the expected factor change may be large. Naturally, it was seen that, in many cases where instruments are applied, a trade-off has to be made between a reduction in risk on the one hand and a decrease in expected performance on the other. However, if the sensitivity to a risk factor is negative (which was the case in the example presented in 
Section 9), a reduction in risk and an increase in performance may go together.

One may wonder whether this approach can also be applied in practice, and how, for example, numerical problems in estimating the sensitivities are solved. Without going into detail we will say something about these problems and their solutions. In Vermeulen (1994) it is described how the sensitivities of Dutch bakeries for various risk factors, can be estimated. To this end, it is assumed that the sensitivities can be explained by firm characteristics. Next, in determining the sensitivities of the bakeries, a data set containing observations of 53 bakeries over 6 years was used. Since the sensitivities were explained by firm characteristics, the data could be pooled and panel techniques could be used to estimate the influence of the characteristics on the sensitivities. Next, also the sensitivities themselves could be computed. The same technique was used to estimate the sensitivities of Dutch manufacturing and retail industries, see respectively Vermeulen et al. (1993) and Vermeulen (1993).

An abundance of similar and related applications exists in performance evaluation (see e.g. Barnea et al. (1975) and Vermeulen et al. (1994), and Spronk and Vermeulen (1994)) and in accounting (see Lev (1980) for example).

\section{Acknowledgements}

The authors gratefully acknowledge the helpful comments of the referees.

\section{References}

Barnea, A., Sadan, S., and Schiff, M. (1975), "Conditional performance review", Management Accounting (November), 19-22.

Berry, M.A., Burmeister, E., and McElroy, M.B. (1988), “'Sorting out risks using known APT-factors", Financial Analyst Journal (March-April), 29-42.

Cooper, D.F., and Chapman, C.B. (1987), Risk Analysis for Large Projects, Models, Methods and Cases, Wiley, New York.
Goedhart, M.H., and Spronk, J. (1991), "Multi-factor financial planning: an outline and illustration", in: A. Lewandowski and V. Vokovich (eds.), Multiobjective Problems of Mathematical Programming, Springer-Verlag, Berlin, 176-199.

Hallerbach, W.G. (1994), "Multi-attribute portfolio selection: A conceptual framework," Ph.D. thesis, Erasmus University Rotterdam, The Netherlands.

Keeney, R., and Raiffa, H. (1976), Decisions with Multiple Objectives, Preferences and Value Trade-offs, Wiley, New York, 1976.

Lev, B. (1980), "On the use of index models in analytical reviews by auditors", Journal of Accounting Research 18/2 (Autumn), 524-550.

LoCascio, S., and Spronk, J. (1992), "Multi-factor models for stock returns and new information", Proceedings of the XVI Amases Conference, Treviso, 459-471.

Ross, S.A. (1976), "The arbitrage theory of capital asset pricing", Journal of Economic Theory 13, 341-360.

Ross, S.A., Westerfield, R.W., and Jaffe, J.F. (1993), Corporate Finance, Irwin, Homewood, IL.

Shapiro, A.C., and Titman, S. (1992), "An integrated approach to corporate risk management", in: J.M. Stern and D.H. Chew Jr. (eds.), The Revolution in Corporate Finance, Second Edition, Blackwell.

Spronk, J., and Vermeulen, E.M. (1994), "Interfirm performance evaluation under uncertainty, a multi-dimensional framework", Report R9414/F of the Rotterdam Institute for Business Economic Studies, Erasmus University Rotterdam, The Netherlands.

Spronk, J., and van der Wijst, D. (1987), "A multi-factor framework for firm evaluation", in: W.G. Hallerbach, F.C.J. Bemelman, J.W.R. Schuit and P.J. van Mierlo (eds.), Finance and Investment, State of the Art 1987, Erasmus University Rotterdam, 317-330.

Van Aalst, P.C., Boender, C.G.E., and van Gijzen, W.J.A. (1993), "Asset liability matching for pension funds: a one period model", Report 9318/F of the Rotterdam Institute for Business Economics, Erasmus University Rotterdam, The Netherlands.

Vermeulen, E.M. (1993), “A multi-factor risk analysis of Dutch retail industries", Tinbergen Institute Research Bulletin 5/3 (December), 239-251.

Vermeulen, E.M. (1994), "Corporate Risk Management: A multi-factor approach," Ph.D. thesis, Erasmus University Rotterdam, The Netherlands.

Vermeulen, E.M., Spronk J., and van der Wijst, D. (1993), "A new approach to firm evaluation", Annals of Operations Research 45, 387-403.

Vermeulen, E.M., Spronk, J., and van der Wijst, D. (1994), "Visualizing interfirm comparison", Omega, International Journal of Management Science, 22/4. 